\title{
Análisis del uso e integración de redes sociales colaborativas en comunidades de aprendizaje de la Universidad de Granada (España) y John Moores de Liverpool (Reino Unido) ${ }^{1}$
}

\section{Using and involvement analysis of social and collaborative network in learning communities of the University of Granada (Spain) and John Moores from Liverpool (United Kingdom)}

\author{
Juan Manuel TRUJILLO TORRES, Inmaculada AZNAR DÍAZ \\ y María Pilar CÁCERES RECHE \\ Universidad de Granada
}

Recibido: Septiembre 2014

Aceptado: Febrero 2015

\begin{abstract}
Resumen
En la actualidad, la integración de redes sociales educativas está adquiriendo un papel fundamental, para la mejora de los procesos de Enseñanza-Aprendizaje (E-A) en el ámbito universitario.

El presente artículo sintetiza las aportaciones más relevantes recogidas a través de un estudio descriptivo, centrado en las percepciones del alumnado mediante la administración de un cuestionario y grupos de discusión, inmersos en la red educativa UGR-John Moores, como experiencia de investigación innovadora en la enseñanza universitaria.

El principal objetivo del estudio es conocer el uso e integración de esta red social y educativa, y valorar las posibilidades del aprendizaje colaborativo, para indagar y mejorar las acciones innovadoras desarrolladas en las nuevas metodologías docentes, abordadas en estas dos instituciones universitarias. Entre las contribuciones más destacables encontramos que la utilización de la red UGR/John Moores ha tenido una gran aceptación entre el alumnado, considerando la plataforma como un elemento facilitador para su aprendizaje, capaz de motivarle, generar y compartir sistemáticamente información, aumentando con todo ello, su entorno personal de aprendizaje (PLE). Sin embargo, es preciso incorporar una serie de mejoras respecto a la planificación docente con la integración de las TIC (acción tutorial), así como actualizar la formación del alumnado en uso de herramientas tecnológicas.
\end{abstract}

Palabras clave: TIC, redes, aprendizaje colaborativo, innovación.

\footnotetext{
${ }^{1}$ Investigación financiada con fondos públicos en concurrencia competitiva y evaluada por ANECA mediante el Secretariado de Innovación Docente de la Universidad de Granada (Código 12-20 /12-90) 


\begin{abstract}
Nowadays, the integration of social and educative network has a big role for improvement of Teaching-Learning (T-L) processes in university context.

The present paper summarizes the most relevant contributions collected by a descriptive study, focused on the student's perceptions through the implementation of a questionnaire and focal groups, immersed in educative network UGR-John Moores, as a researching experience of innovation in university.

Knowing the use and integration of this social and educative network and valuing the possibilities of collaborative learning is the main purpose of this study, in order to research and improve the innovative actions developed through new teaching methodologies in both universities.

Some of the most relevant contributions refer to the impact that UGR/John Moore network has got in reference to a good acceptance between students, considering it, as an element that enables their learning, promotes their motivation and produces and shares information systematically; increasing their personal learning environment (PLE).

However, it is necessary to incorporate different sets of improvements in relation to teaching planning with the use of ICTs (tutorial actions); as well as, update a specific training of students in the use of technology tools.
\end{abstract}

Keywords: ICT, network, collaborative learning, innovation.

Internet es una realidad ya asentada en nuestro entorno social, que unida a la constante aparición de tecnologías emergentes vienen a incorporar nuevas posibilidades de uso e integración didáctico-pedagógica en nuestro sistema educativo. Respecto al ámbito universitario, este reconocimiento de integración para la mejora de la calidad, se está llevando a cabo en los procesos de enseñanza-aprendizaje convirtiéndose en un hecho constatable por diversos autores y estudios (Laurillard, 2010; Trujillo, Cáceres, Hinojo \& Aznar, 2011; Marín, Vázquez \& Cabero, 2013; Gea \& Montes, 2013; Cabero \& Marín, 2014).

Las redes sociales colaborativas son una oportunidad para potenciar el aprendizaje activo, las experiencias compartidas, el compromiso de docente y discentes, etc. Así, aunque las redes sociales surgieron como sistema para relacionar y vincular a los individuos, su presencia en las organizaciones, y por ende la educativa, es cada día más importante, siendo tendencia definitoria de la actual fase de desarrollo de Internet, pues el carácter potencial, comunicativo y relacional que irradia el desarrollo de las mismas está sobradamente justificado. Las redes sociales pues, se hacen masivas, presentando un funcionamiento de flujo basado en la participación, el cambio de actitud y el compromiso activo realizando funciones de vinculación social que se basan en el conjunto y en la inteligencia colectiva. Con ello, se han multiplicado enormemente las posibilidades para la educación, la comunicación, la difusión y la acción social pues, en definitiva, se trata de una nueva táctica de la acción social colectiva. Por este motivo, es preciso comprender su lógica de funcionamiento y las interacciones establecidas entre los componentes para tener una visión de lo que sucede en el mundo más cercano (Trujillo, 2013). 
Estas redes son un principio de construcción de interactividad, de un cambio de rol del docente y del alumnado, de promoción y creación de espacios compartidos de conocimiento mutuo, el desarrollo de la confianza, pertenencia y compromiso y, en definitiva, la creación de una verdadera comunidad de aprendizaje que valore la implicación de todo el colectivo y la necesidad del grupo para la promoción y desarrollo pleno de la individualidad.

En este sentido, trabajamos en los últimos años en diferentes proyectos de Investigación e Innovación Docente relacionados con las TIC aplicados a la docencia, y más concretamente en el aprendizaje cooperativo en entornos virtuales. En las últimas convocatorias del Secretariado de Innovación Docente de la Universidad de Granada, han sido subvencionados los siguientes proyectos: El aprendizaje cooperativo en entornos virtuales para la adquisición de competencias de los futuros docentes en el marco de convergencia europeo (2008), Competencias de los futuros docentes en el Espacio Europeo de Educación Superior: trabajo colaborativo en espacios virtuales (2008), El Cross Age per tutoring como estrategia didáctica para el desarrollo del liderazgo educativo en la formación docente (2009), El método por proyectos como acción innovadora para la formación docente mediante el aprendizaje entre iguales (2010), Posibilidades de aplicación educativa de herramientas web 2.0 y cambio metodológico en el proceso de convergencia europeo (2010), La adquisición de competencias mediante el método por proyectos y las herramientas web 2.0 como estrategias didácticas innovadoras en la formación docente universitaria (2011), El Elearning en contextos de aprendizaje colaborativo e interdisciplinar como recursos didácticos innovadores para el desarrollo profesional docente en la enseñanza universitaria (2012), El b-learning como propuesta didáctica innovadora para la formación de competencias docentes en contextos de aprendizaje colaborativos e interuniversitarios (2013).

Se nos presentan pues una serie de retos atendiendo a una sociedad cada vez más global y cambiante, donde la organización escolar y los procesos de enseñanzaaprendizaje necesitan incorporar una serie de transformaciones significativas. Precisamos, consecuentemente, de un profesor abierto al conocimiento y eminentemente innovador, capaz de adquirir una mayor autonomía y responsabilidad estratégica, promotor de valores y potenciador/desarrollador de redes de trabajo comunitario.

Estos proyectos de Innovación Docente (2008-2013) siempre han pretendido, desde el cambio de actitud, la participación colectiva y la interacción como eje básico de su funcionamiento. Potenciando lo que De Haro $(2010,29-35)$ expone como factores para el trabajo red colaborativo y una educación 2.0: actitudes que caracterizan la cooperación en la sociedad del conocimiento (altruismo, colaboración y respeto), habilidades o capacidades que deben desarrollar los alumnos (gestionar el propio conocimiento, pensamiento creativo y pensamiento crítico) y competencias que deben adquirir para desenvolverse en dicha sociedad (planificación para la investigación generando resultados, conocimiento de los medios para la colaboración, producción de objetos digitales, importancia de la comunicación para la información y la creación de conocimiento conjunto). Las redes sociales pues, se conforman con individuos y sus relaciones (Harada, Lum \& Souza, 2003; Kimble, Hildreth \& Bourdon, 2008; Rodríguez 
Illera, 2008; Read, 2013). Estas relaciones dan sentido a las dinámicas de trabajo conjunto que se establecen materializando vínculos entre los miembros que constituyen una gran red, en la que cada uno de sus integrantes se encuentra relacionado, en mayor o menor medida, con todos y cada uno de los miembros que la forman. Una estructura nodal que enriquece, sin duda, el poder del acto comunicativo.

No hay duda alguna de que una de las señas de identidad más valoradas en los procesos de innovación pedagógica, es el poder del acto comunicativo. Aquel que genera un entorno propicio para la interacción, donde es fácil cuestionar y responder, construir y concluir conocimiento, identificarse con el grupo, etc. Y es que la construcción activa precisa del compromiso para el diálogo conformando un espacio multidireccional de relaciones de pensamiento que observan en la palabra su vehículo conductor y primario. Dado que la comunicación es uno de los procesos humanos con una mayor presencia en su desarrollo social, no es arriesgado adelantar que el futuro está repleto de posibilidades con relación a la enseñanza. Si los sistemas de formación en red han de tener algún rasgo diferencial es el trabajo colaborativo y la multiculturalidad (Trujillo, 2013:100).

\section{Gestión del conocimiento con redes sociales. Conectarse para el aprendizaje y el desarrollo competencial}

La comunicación mediante Internet y la puesta en común de un grupo de personas que trabajan en torno a proyectos compartidos permite el desarrollo del pensamiento crítico, el auto-aprendizaje, reconocimiento expreso de la diversidad, desarrollo de la competencia digital entre otras, el potencial investigador a través de proyectos y estudios de caso, etc. (Alonso \& López, 2008). Esta propuesta metodológica conlleva en sí misma una nueva forma de proceder, más motivadora y dinámica, interactiva y significativa, que sin duda cambia la perspectiva tradicional de la capacitación y el desarrollo profesional. En este nuevo contexto, las redes educativas parecen posicionarse como protagonistas para el desarrollo de las diferentes competencias de ejecución en nuestro alumnado. En cualquier caso nunca había habido tantas posibilidades ciertas para acceder a la información y poner en común de manera interdisciplinar al colectivo. Las herramientas de apoyo para ello, fundamentadas y apoyadas en Internet, se conforman como la base tecnológica de la forma organizativa que caracteriza la era de la información. Y es que está cambiando la manera de acceder a esta información, la toma de decisiones de acceso, la integración de medios, la multiplicidad de lenguajes, la ubicuidad, la interactividad, la calidad técnica (Jubany, 2012:28).

Encontramos en el momento actual, en el campo de la educación, un cambio de actitud enfocado hacia el valor del colectivo en la gestión del conocimiento. Algunos ejemplos de ello lo confirman: GrinUGR, EducaconTIC, Aulablog, Edublog, Octeto, Internet en el aula, EduCAT2.0, Dim Global, etc. De igual modo, surgen proyectos colaborativos abiertos a la participación de la ciudadanía que justifican la importancia del aprendizaje invisible (Cobo \& Moravec, 2011) y la relevancia para la educación y el desarrollo de competencias desde los ámbitos no formal e informal donde se cumple el propósito y el valor de la inteligencia de la multitud (Gutiérrez-Rubí \& Freire, 2013). Ejemplos de ello son MedialabPrado, El campo de Cebada, OpenStreetMap, MakeSpace, 
Goteo, Tabacalera.net, etc.

Así pues, la conformación de redes educativas revelan comunicación multidireccional que apuesta por compartir conocimientos, el sentimiento de comunidad que ayudan a integrar a personas con aspectos de diversa índole comunes y cooperación, principalmente desinteresada, la cual es capaz de crear en, con y para la colectividad (Trujillo, 2013). En el grupo parece evidente que hay más inteligencia que en el potencial de los individuos considerados de manera aislada y es que todo individuo puede aportar algo y del mismo modo, todos ignoramos cuestiones por lo que precisamos de esa generación de relaciones interconectadas para aprender y construir gestionando conocimiento. Desconocemos mucho más de lo que permanece en nosotros significativo por lo que aprender, descubrir e innovar debe ser un placer en cada momento. En definitiva, lo que promueven estos nodos es la materialización de los vínculos entre miembros para constituir una gran red en la que cada uno de sus componentes y elementos se encuentra de alguna forma vinculado con la totalidad de los otros integrantes.

Con ello, podemos mirar las redes sociales en la educación, desde al menos tres perspectivas educativas complementarias (Castañeda \& Gutiérrez, 2010): aprender con redes sociales; aprender a través de redes sociales y aprender a vivir en un mundo de redes sociales. La realidad nos muestra, sin duda, la presencia del alumnado en las redes sociales y es preciso aprovechar desde el ámbito educativo dichos espacios de interacción y comunicación. Desde el ámbito formal, por supuesto, pero también haciendo hincapié en la necesidad de vehicular los aprendizajes informales y no formales poniéndolos en común y gestionando de manera efectiva el conocimiento que se advierte en los mismos. Los componentes de la red estarán informados y tendrán oportunidad de aprender gracias a dicho trabajo colaborativo apareciendo usuarios activos y otros pasivos, pero igual de necesarios en la propia dinámica de funcionamiento interno. Es preciso, adquirir una identidad digital que valore ciertamente las posibilidades de un mundo conectado. Las redes sociales pues, se conforman con individuos y sus relaciones. Estas, en última instancia, de calidad y que dan sentido a las dinámicas de trabajo conjunto que se generan. Lo evidenciamos en Internet con la presencia de multitud de espacios que se configuran en torno a una estructura relacional nodal entre sus miembros y todo ello gracias a una temática de interés (imágenes, vídeos, intereses, investigación, etc.) que los une en último lugar podemos hablar ciertamente de conexiones puesto que aunque las relaciones no sean en muchas ocasiones directas sí lo están en todo momento de manera indirecta.

En este sentido y siguiendo a Camacho (2010), podemos afirmar que las redes sociales colaborativas en el aula promueven la ampliación de fronteras, facilitación de la comunicación, construcción compartida del conocimiento, cambio de rol de los recursos, potencian la motivación favoreciendo la actitud abierta y la capacidad de relación, generan comunidades de aprendizaje efectivas, mejoran la gestión organizativa y facilitan la metacognición. Aprendemos con los demás desde la generación de un entorno de trabajo colaborativo estable, gestionando y accediendo al conocimiento de manera inmediata, promoviendo la actualización permanente y ampliando la base de nuestro aprendizaje. 
Al mismo tiempo, Martínez \& Solano (2010) señalan la importancia que tiene en la enseñanza virtual y sistemas de formación en red los rasgos diferenciales del trabajo colaborativo y la multiculturalidad. Lo que nos lleva a plantear que esta nuevo contexto de aprendizaje supone una oportunidad para generar espacios educativos de gran valor y utilidad para el diseño de nuevas metodologías didácticas. Los centros educativos se ven obligados a plantearse modificaciones en los procesos (formación continua), en el objeto de la enseñanza (alfabetización digital), en los objetivos educativos (nuevas capacidades y conocimientos), en el organigrama (infraestructuras, equipos, gestión, etc.), en el rol del profesor (facilitador, mediador) y en el del alumno (aprender a aprender) y, por último, cambios en los contenidos didácticos (más información, interactividad, convergencia de lenguajes, etc.).

Una enseñanza basada en la recepción pasiva de la información y el uso indiscriminado de la memoria puede conllevar la falta de compromiso e implicación consiguiente pues, de este modo, el alumnado no encuentra compromiso de implicación en el proceso, vinculación a su mundo real y las problemáticas que se derivan de él, atención a la diversidad de intereses y competencias, entre otros, lo que supondría condenar al fracaso a los procesos de Enseñanza-Aprendizaje (E-A) que así lo establezcan. Los procesos de E-A han de suponer toma de decisiones, liderazgo distribuido, proceso investigador, indagación y reflexión compartida, entre otras (Hinojo-Lucena \& Fernández, 2002; Solano, 2006; Palloff \& Bratt, 2007; Thelwal, 2008; Trujillo, Cáceres, Hinojo-Lucena \& Aznar, 2009).

Esto, que aparentemente parece sencillo, no lo es. La tarea multidisciplinar a largo plazo requiere de una planificación metódica que ha de caracterizarse por la implementación del método científico y el uso de metodologías activas. Este tipo de aprendizaje basado en proyectos y problemas, que prepara al alumno para la solución de cuestiones que son significativas para su vida, es una oportunidad para potenciar el trabajo en colaboración con los otros (Trujillo, 2013). Con ello, el aprendizaje a través de la redes sociales encuentra su justificación en las teorías del aprendizaje que defiende la importancia del aprendizaje social, el aprendizaje colaborativo, el conectivismo, la educación expandida y los entornos personales de aprendizaje (PLE) (García-Valcárcel, 2013). Aprender y conocer toman como base la diversidad, desde la creación de nodos interrelacionados donde la capacidad del alumnado para establecer conexiones es clave. La habilidad para estar en predisposición para instruir el contenido del futuro se revela como más importante que el saber propio del presente. Formación permanente autogestionada para generar redes distribuidas de conocimiento y que al mismo tiempo se han de convertir en comunidades de aprendizaje dinámicas.

El protagonismo y dominio de las TIC por parte de los usuarios está hoy más que nunca presente y en la conformación de redes sociales colaborativas emergen nuevas tácticas de la acción social colectiva. Aspectos que es importante considerar, en este sentido, serían la formación de la conciencia de los intereses, la confianza como factor decisivo, comunidad, comunicación y cooperación. 


\section{La red UGR - John Moores. Conectarse para aprender}

Las metodologías activas, consecuentemente, son la clave de esta renovación pedagógica, pues se conforman como fundamento de estos procesos de intercambio, resolución colaborativa y construcción de conocimiento conjunto. Y todo ello desde una estructura interactiva para con el entorno y su lógico compromiso en lo referente a la intervención social, aprendiendo de y con los demás.

De este modo, las redes sociales educativas, evidenciamos, presentan estas características definitorias y se posicionan pues con la idea de potenciar y facilitar los procesos comunicativos, con la lógica de ampliar fronteras y espacios de conocimiento. Se promueven estructuras, desde la base, para el trabajo colaborativo favoreciendo la actitud positiva y abierta y la competencia relacional. Presentan mecanismos de motivación reforzando y mejorando el sentido de pertenencia a través de la gestión interna y el ejercicio de un liderazgo distribuido, resiliente y compartido. Del mismo modo, se mejoran procesos metacognitivos tomando conciencia de la relevancia del aprendizaje social.

Teniendo en cuenta los usuarios potenciales y la motivación hacia las redes sociales, el uso educativo puede centrarse en los proyectos de aula, en el portafolio de la asignatura, en los proyectos intercentros, las comunidades virtuales de aprendizaje o las comunidades de prácticas docentes (Comer, 1993; Cáceres \& Aznar, 2005; Aznar, Cáceres \& Hinojo, 2005; Trujillo \& Hinojo-Lucena, 2011; Marqués, 2011; Valverde, 2011; De-Gouveia, 2012; Maiz \& Tejada, 2013). Estos mismos autores, reseñan destrezas de razonamiento que favorecen la transformación de la información en conocimiento y que hemos intentado llevar a cabo en la red social educativa UGR-John Moores (http://ugrliverpool.mixxt.com/) en colaboración con la Universidad de Granada (España) (UGR) y la Universidad John Moores de Liverpool (Reino Unido): organización de la información; establecimiento de relaciones mentales de lo que se conoce; análisis documental desde diferentes perspectivas y/o enfoques; síntesis del material recopilado; tarea de inferir y deducir.

Entre los objetivos propuestos para el desarrollo de la plataforma y consecuentemente la mejora del proceso de enseñanza-aprendizaje, a través del trabajo colaborativo en red tenemos los siguientes:

- conformar una red educativa de desarrollo profesional y personal en la que todos sus elementos constituyentes sean partícipes de los procesos de gestión del conocimiento;

- mejorar competencialmente en base al desarrollo de aprendizajes basados en proyectos y estudios de caso;

- utilizar herramientas de la web social e integrarlas efectivamente;

- comprender y valorar significativamente la necesidad de aprender a aprender y verificar la relevancia de la competencia emocional; 
- valorar la red UGR-John Moores como una oportunidad para la innovación del currículo, la interdisciplinariedad, la transformación educativo-social y la transferencia y gestión efectiva de conocimiento.

En definitiva, se trata de operar motivacionalmente desde los intereses de los discentes, pues la relación directa con la praxis suele presentarse en la mayoría de los casos y los aprendizajes revisten secuencias graduales de conocimiento permanente y situado. Conocimiento, colaboración, innovación, participación, ética, compartición, creación compartida, red, organización plana, comunidad, etc., son sus valores y formas de actuar. "No hay duda alguna de que una de las señas de identidad más valoradas en los procesos de innovación pedagógica es el poder del acto comunicativo. Aquel que genera un entorno propicio para la interacción, donde es fácil cuestionar y responder, construir y concluir conocimiento, identificarse con el grupo, etc. Y es que la construcción activa precisa del compromiso para el diálogo conformando un espacio multidireccional de relaciones de pensamiento que observan en la palabra su vehículo conductor y primario.

Por ello, precisamos de la figura de un profesorado y alumnado que faciliten el diálogo, la capacidad para interactuar, que no presente patrones rígidos en su actuación dentro y fuera del aula, que comprendan que todos pueden enseñar y siempre colectivamente las aportaciones son de mayor calidad, donde se facilite el intercambio experiencial, , que haga fluir el discurso como método de actuación para la generalización, socialización e interiorización de su uso, que en definitiva construyan un ambiente que otorgue el derecho a la palabra y justifique el gozo por compartirla. Las redes sociales son una oportunidad para ello” (Trujillo, 2013:100).

\section{Método}

\section{Propósito}

La investigación que se presenta es parte de otra de mayor calado y proyección internacional, entre instituciones de educación superior y del ámbito universitario de España, Portugal y Reino Unido, en relación al uso y aprovechamiento de las redes sociales en el ámbito educativo, aplicada al mejoramiento de la enseñanza virtual mediante entornos de aprendizaje colaborativos. En concreto, el propósito de esta parte del estudio ha consistido en analizar, describir y valorar por parte del alumnado universitario, el uso e integración de la red social educativa UGR-Liverpool John Moores para la mejora de los procesos de Enseñanza y Aprendizaje (E-A) y las posibilidades que para el aprendizaje no formal presentan.

\section{Muestra}

La muestra de este estudio estuvo compuesta por una selección de 87 alumnos que cursan estudios universitarios en las Facultades de Educación, tanto de la Universidad de Granada como de la Universidad John Moores de Liverpool. En concreto, todo el alumnado participante en dicho estudio formo parte de proyectos de innovación 
docente universitaria en la materia de Organización de Centros Educativos, perteneciendo a las titulaciones de Grado en Educación Primaria, Grado en Educación Infantil y el Grado en Pedagogía. Se realizó un muestreo aleatorio simple entre toda la población de estudiantes, siendo la muestra aceptante y productora de datos 83 sujetos en total (31 mujeres, 52 hombres, 22 alumnos JML y 61 alumnos UGR).

\section{Instrumentos}

Dado el carácter descriptivo de la investigación, se utilizó un método de Encuesta, optándose por la elaboración de un cuestionario " $a d$ hoc", realizándose en dos fases diferenciadas y haciéndolas coincidir cronológicamente con la administración del cuestionario. De esta forma, para la parte cuantitativa, el cuestionario se estructuró en bloques y/o dimensiones claramente diferenciadas (expectativas del alumnado, uso y aprovechamiento de las TICs y valoración/satisfacción), compuesto por un total de 66 ítems cerrados, de tipo Likert, de 4 alternativas (1. CD: Completamente en Desacuerdo, 2. D: Desacuerdo, 3. A: De Acuerdo y 4.CA: Completamente de Acuerdo). La aplicación del cuestionario se realizó online.

Se aplicó la prueba de Fiabilidad Alfa de Cronbach para determinar el grado de consistencia interna del instrumento, dando como resultado 0,867 , considerada alta por la comunidad científica.

Los resultados de $\alpha=0,867$ en la prueba Alfa de Cronbach y de 0,842 en la prueba Dos mitades de Guttman son considerados muy buenos, indicando una muy alta fiabilidad del instrumento (precisión alta).

Respecto a la validez de los instrumentos se ha seguido la técnica de "juicio de expertos” (validez de contenido). Igualmente se realizó una validez semántica mediante una representatividad, relevancia y plausibilidad de los datos, contrastando diversas perspectivas de significación (de contenido con distintas personas y de instrumentos utilizando diferentes procedimientos para la recogida de información). Dicha validez se realizó por profesionales de la Universidad John Moores de Liverpool produciéndose algunas variaciones en ítems y supresiones.

Para la parte cualitativa de la investigación, se utilizaron los grupos de discusión con participación de informantes clave: cuatro alumnos, dos profesores y coordinador/moderador (para la selección del alumnado y profesorado se optó por un muestreo criterial en función de experiencia previa en los proyectos de innovación docente universitaria), y los propios foros y chats de los blogs y de la plataforma utilizada, complementando la información del cuestionario, y dando un poco más de luz y comprensión a los resultados cuantitativos. Para el vaciado y análisis de la información relativa a foros y chat se realizó de forma manual y en algún caso en concreto se utilizó la aplicación informática Snapchat. 


\section{Diseño y Procedimiento}

Este estudio surge como fruto del análisis sistemático de necesidades llevado a cabo durante los cursos 2008/2013 en base a aspectos relacionados con la metodología blended eLearning y entornos de enseñanza virtuales. Se desarrolló en dos fases diferenciadas: análisis de necesidades, para conocer y priorizar las necesidades de la muestra, y en una segunda fase de evaluación y toma de decisiones, para determinar la incidencia de dichas necesidades. Se tomó como referencia las materias relacionadas con Organización/Gestión/Administración de Centros Educativos del grado de maestro de Primaria, impartidas por ambas instituciones universitarias durante el curso 2012/2013. Al comienzo de dichas materias, se pasó un cuestionario al alumnado que constaba de 29 ítems de diverso tipo, varias preguntas abiertas y 15 ítems cerrados que corresponden a una escala de valoración tipo Likert de cuatro opciones para indicar niveles de conocimiento o capacitación. Una vez finalizados los contenidos de dicha materia mediante entornos de aprendizaje colaborativo virtuales, se realiza una segunda pasación, incluyendo los comentarios y anotaciones de la plataforma y demás herramientas online y grupos creados al efecto en diferentes redes sociales, analizándose posteriormente en varias reuniones/seminarios de seguimiento.

El diseño metodológico es eminentemente descriptivo, no experimental y no correlacional, siendo el método de encuesta utilizado en sus distintas vertientes (cuestionario, focus group y puestas en común de información mediante plataforma).

Se realizaron análisis estadísticos descriptivos (medidas de tendencia central de la muestra), análisis inferencial mediante pruebas no paramétricas (chi-cuadrado de Pearson), fijándose un nivel de confianza del 95\% y análisis factorial (tabla 2 y 3) Para dichos análisis estadísticos se ha utilizado el paquete estadístico SPSS versión 19.

Dado que se trata de un estudio exploratorio y descriptivo, se optó por la realización de un análisis factorial que permitiese detectar que ámbitos destacan y podrían agruparse entorno a otros factores que determinaran un nuevo y mejorado instrumento de evaluación definitivo. Antes de realizar el análisis factorial empleamos el test de esfericidad de Barlett, que ha revelado un Chi-cuadrado aproximado de 436,52, 45 gl., significativo a .000; y el índice de KMO de Káiser-Mayer-Olkin de 0,720 considerándose bueno (Martín, 1999). Esta información nos ha permitido tomar la decisión de realizar el correspondiente análisis factorial, utilizándose el método de Análisis de los Componentes Principales (PCA), con rotación de variables, criterio "Varimax" con Kaiser. Pasamos a presentar los resultados obtenidos:

Se presenta igualmente la tabla de varianza donde, siguiendo el método de componentes principales, explica la variabilidad total por cada componente y la que explica cada uno. En total 4 factores explican el cuestionario, 10 variables, con un $57,509 \%$ del total de la varianza, porcentaje considerado como aceptable teniendo en cuenta el número de variables seleccionadas. 


\begin{tabular}{|c|c|c|c|c|c|c|c|c|c|}
\hline \multirow{2}{*}{ 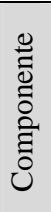 } & \multicolumn{3}{|c|}{ Autovalores iniciales } & \multicolumn{3}{|c|}{$\begin{array}{l}\text { Sumas de las saturaciones al } \\
\text { cuadrado de la extracción }\end{array}$} & \multicolumn{3}{|c|}{$\begin{array}{l}\text { Suma de las saturaciones al } \\
\text { cuadrado de la rotación }\end{array}$} \\
\hline & Total & $\begin{array}{c}\% \\
\text { varianza }\end{array}$ & $\begin{array}{c}\% \\
\text { Acumu- } \\
\text { lado }\end{array}$ & Total & $\begin{array}{c}\% \\
\text { varianza }\end{array}$ & $\begin{array}{c}\% \\
\text { acumu- } \\
\text { lado }\end{array}$ & Total & $\begin{array}{c}\% \\
\text { varianza }\end{array}$ & $\begin{array}{c}\% \\
\text { acumu- } \\
\text { lado }\end{array}$ \\
\hline 1 & 2.258 & 22.578 & 22.578 & 2.258 & 22.578 & 22.578 & 1.687 & 16.869 & 16.869 \\
\hline 2 & 1.308 & 13.077 & 35.655 & 1.308 & 13.077 & 35.655 & 1.608 & 16.083 & 32.952 \\
\hline 3 & 1.125 & 11.252 & 46.907 & 1.125 & 11.252 & 46.907 & 1.343 & 13.431 & 46.383 \\
\hline 4 & 1.060 & 10.602 & 57.509 & 1.060 & 10.602 & 57.509 & 1.113 & 11.126 & 57.509 \\
\hline 5 & .985 & 9.848 & 67.357 & & & & & & \\
\hline 6 & .894 & 8.936 & 76.293 & & & & & & \\
\hline 7 & .861 & 8.608 & 84.900 & & & & & & \\
\hline 8 & .688 & 6.880 & 91.781 & & & & & & \\
\hline 9 & .467 & 4.671 & 96.452 & & & & & & \\
\hline 10 & .355 & 3.548 & 100.000 & & & & & & \\
\hline
\end{tabular}

Tabla 1. Análisis Factorial. Método de extracción: Análisis de componentes principales.

Factor 1. "Formación en TIC y redes sociales". Factor 2. "Utilización y uso de plataforma de aprendizaje". Factor 3. "Expectativas Comunidades de aprendizaje" y Factor 4. "Valoración trabajo colaborativo y tutorías virtuales".

\section{Resultados}

A continuación se presenta, de forma gráfica, algunos de los resultados más relevantes en relación al objeto de estudio:

En general, los resultados confirman que un alto porcentaje del alumnado no ha tenido problemas asociados con el uso de la plataforma colaborativa (69\%). Cuando las dificultades aparecen, éstas vienen relacionadas principalmente con el alta en la plataforma (43.9\%) y el dominio y uso general de la red Internet (31.4\%).

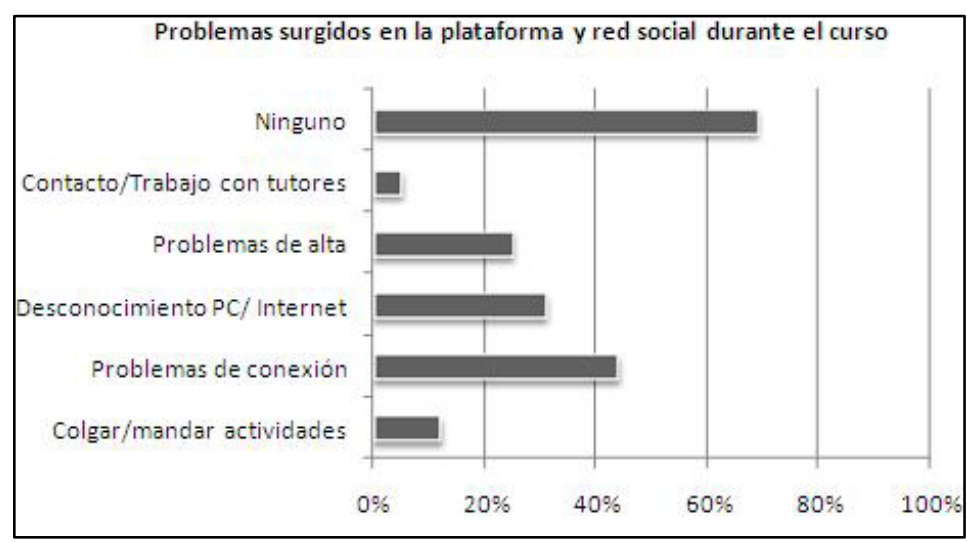

Gráfico 1. Problemas surgidos en la utilización de la Plataforma y red social durante el curso. 
En relación a la valoración del alumnado respecto del aprendizaje emanado de la red social colaborativa, encontramos que prácticamente la totalidad (95.5\%) lo valoran positivamente. Estos datos evidencian el valor intrínseco de la Red social colaborativa para construir relaciones y aprendizaje.

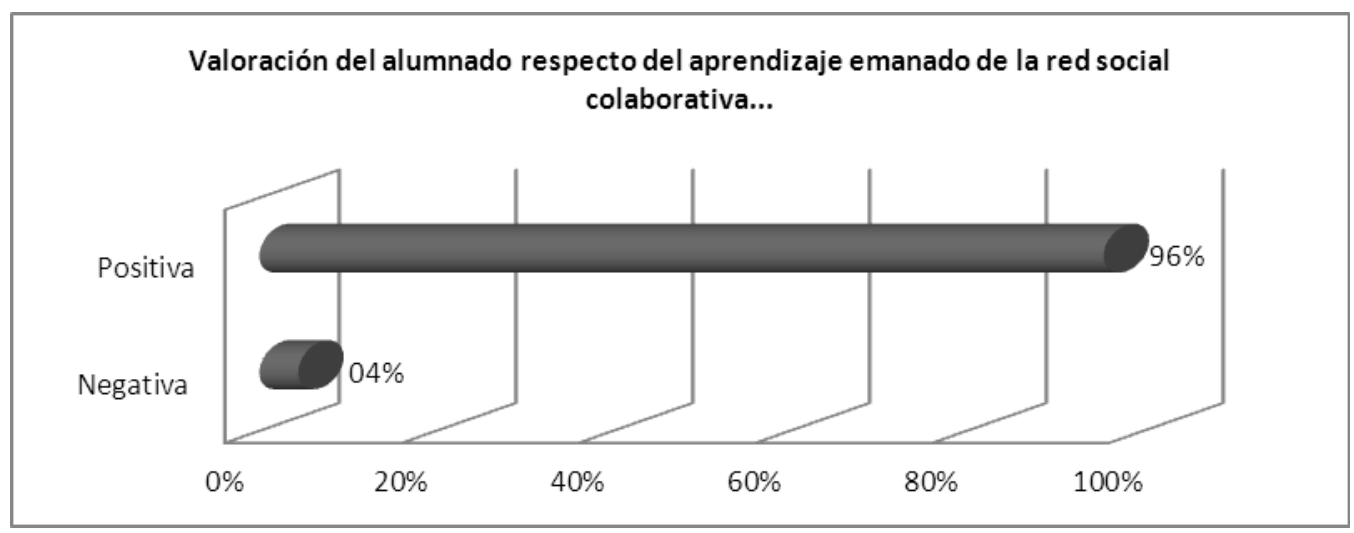

Gráfico 2. Valoración de la red respecto del aprendizaje en la Red.

Se advierte, del mismo modo y con facilidad, que la satisfacción del alumnado con respecto al uso de la Red colaborativa es muy alta (77.1\% muy satisfecho y $20.2 \%$ satisfecho). No apareciendo ninguna persona muy insatisfecha y tan solo el $0.6 \%$ insatisfecho. Los resultados confirman la excelente aceptación, por parte del alumnado, hacia el trabajo colaborativo red y en comunidad y su motivación respecto del uso de plataformas virtuales.

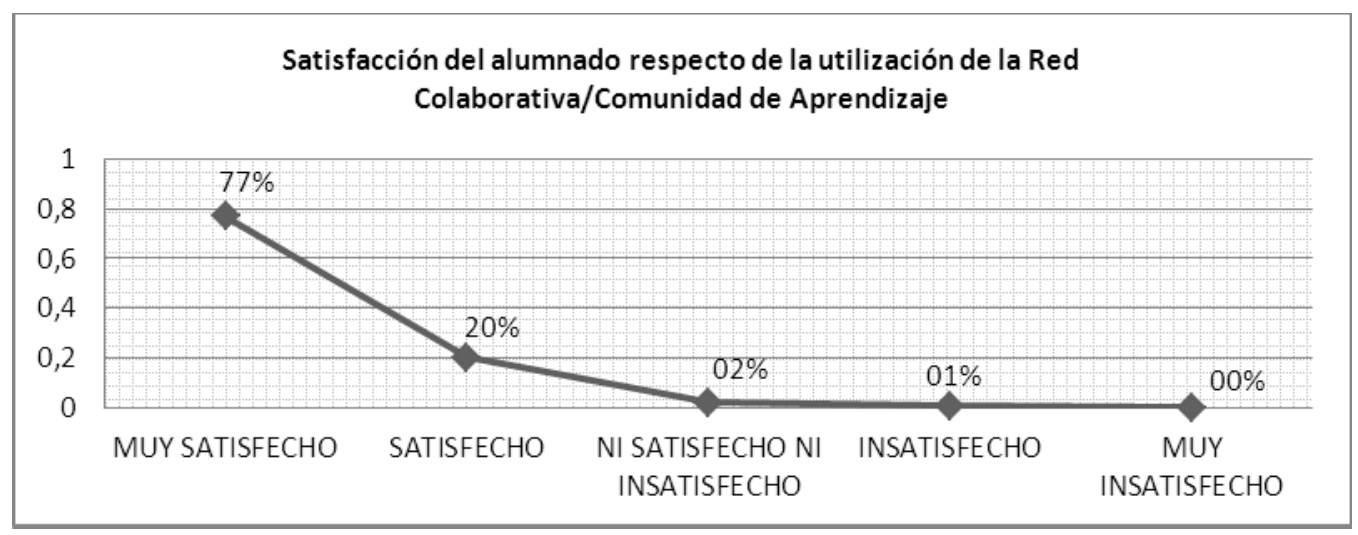

Gráfico 3. Satisfacción del alumnado respecto del uso de la Red. 
Esta dimensión evidencia un grado de satisfacción, con respecto a la acción tutorial desarrollada, muy buena y buena (57\% / 14\%). Tan solo un $29 \%$ ha opinado como regular dicho desempeño. Con ello, aunque los resultados son muy positivos quizás habría que incidir y hacer hincapié en una mejor planificación de dicha acción tutorial en entornos virtuales.

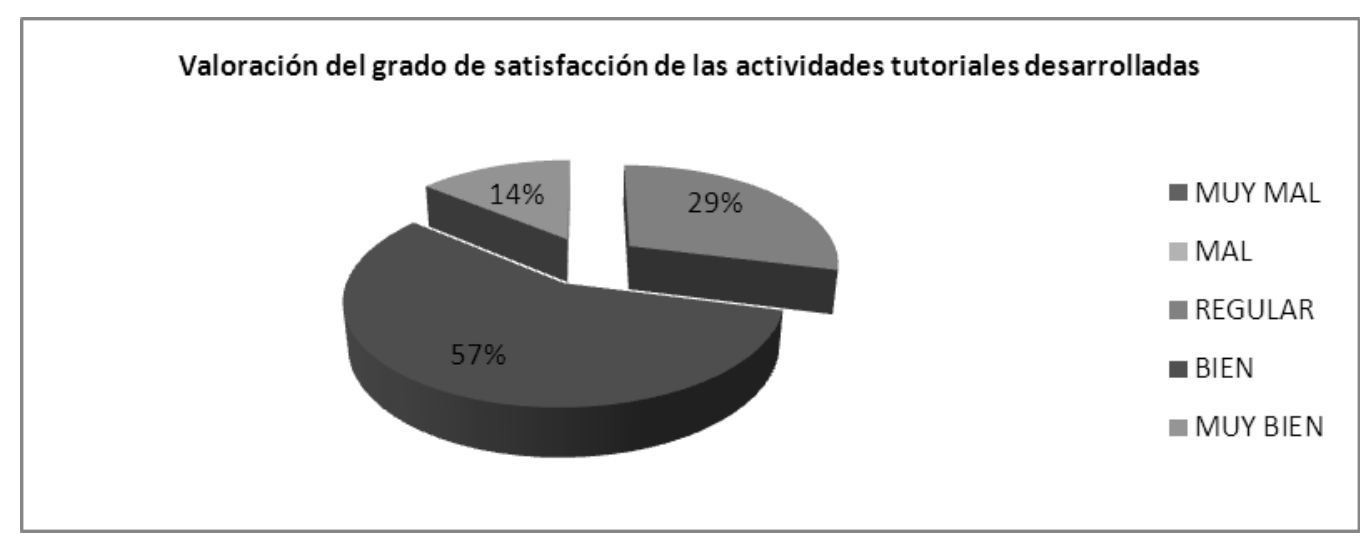

Gráfico 4. Valoración satisfacción actividades tutoriales.

En la siguiente gráfica se puede apreciar la puntuación obtenida en el cuestionario, cuyo rango oscila entre 1 (menor) y 5 (mayor), de los siguientes aspectos analizados:

1. Adecuación de la información de la plataforma y/o red comunitaria de aprendizaje.

2. Idoneidad de los medios y recursos utilizados para la consecución de las metas establecidas.

3. Interacción entre los participantes/miembros de la comunidad de aprendizaje.

4. Planificación horaria del alumnado a la actividades grupales/seminarios.

5. Grado de dedicación e implicación de asesores externos.

6. Intercambio de información y trabajo cooperativo entre compañeros.

7. Valoración del profesorado participante (tutores).

8. Interés por los contenidos y aplicabilidad al desarrollo docente futuro.

9. Valoración general del flujo de información y aportación para el aprendizaje de la red UGR/John Moores. 


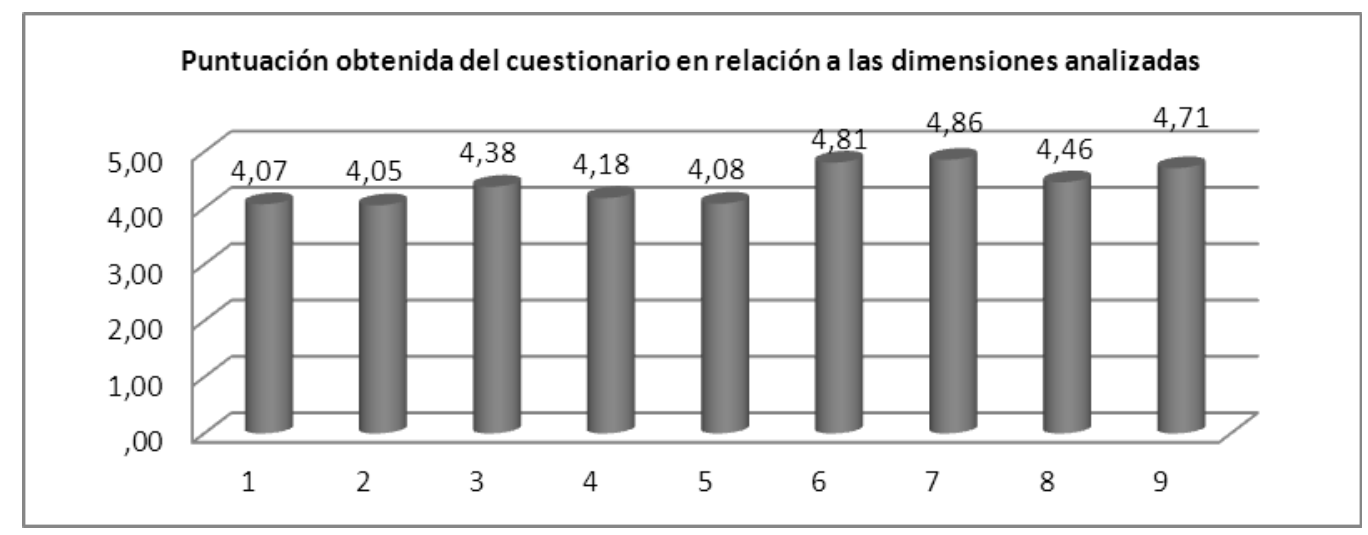

Gráfico 5. Rangos de puntuación de dimensiones del cuestionario.

A tenor de los datos recogidos, detectamos una valoración positiva de la información que la plataforma ofrece (4.07) y la interacción promovida entre los miembros participantes (4.38). Destacan las percepciones sobre el trabajo colaborativo y su relevancia en los procesos de E-A y la valoración del profesorado participante (4.81 / 4.86). También es preciso destacar el interés por los contenidos expuestos en la plataforma y expresamente la determinación sobre la aplicabilidad futura de dichos contenidos (4.46). Los datos, de igual modo, muy positivos en cuanto a la implicación de asesorías externas (4.08) que potencian la constitución de reales y efectivas comunidades de aprendizaje y la idoneidad de recursos y medios utilizados para el desarrollo de competencias y cumplimiento consecuente de objetivos (4.05).

Por otro lado, los alumnos consideran que uso de las TIC en la formación en la Universidad es realmente importante ya que la gran mayoría de las respuestas (99\%) están totalmente de acuerdo o de acuerdo con la afirmación.

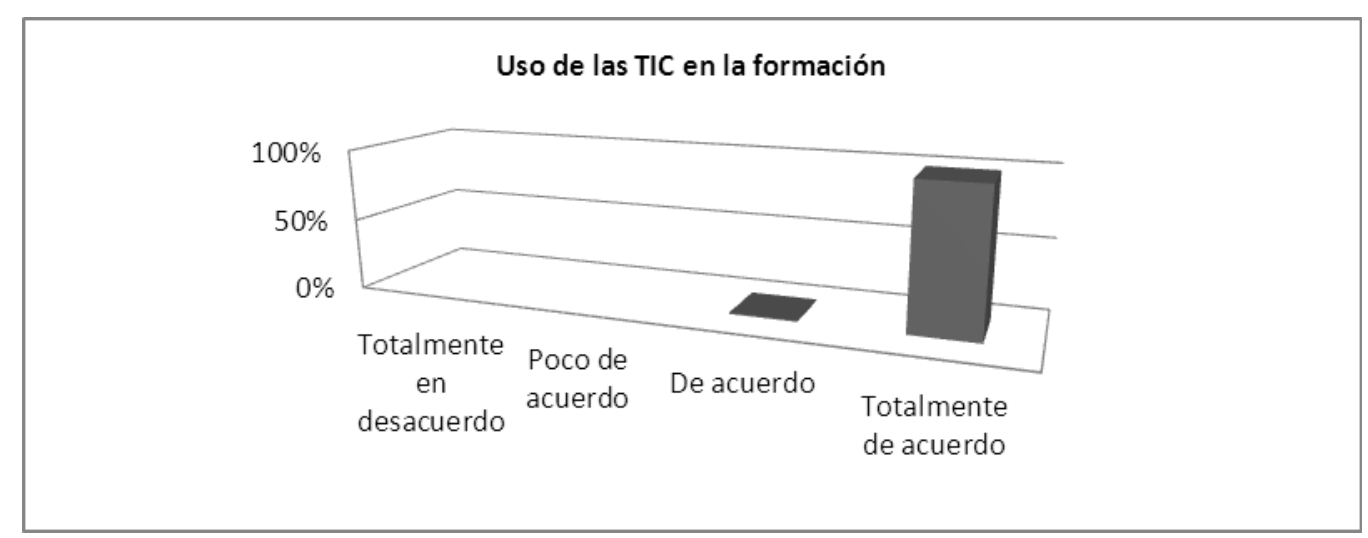

Gráfico 6. Uso de las TIC en la formación en la Universidad. 
La eliminación de las distancias y la posibilidad de conocer personas distintas, como valor principal de las experiencias que nos provocan las nuevas tecnologías es un aspecto muy importante para nuestro alumnado, ya que el 77\% de ellos así acuerdan.

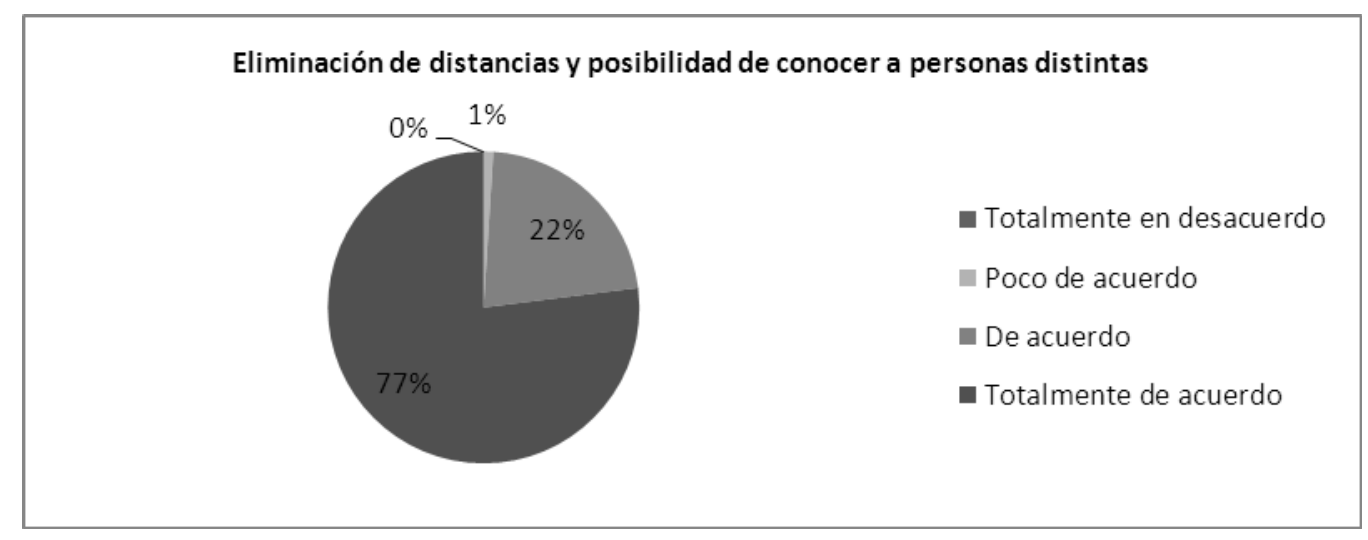

Gráfico 7. Eliminación de distancias y conocer personas.

Otra de las principales ventajas de la utilización de la red colaborativa es la flexibilidad para aprender en el tiempo y en el espacio, esta afirmación es compartida por el $100 \%$ de nuestro alumnado.

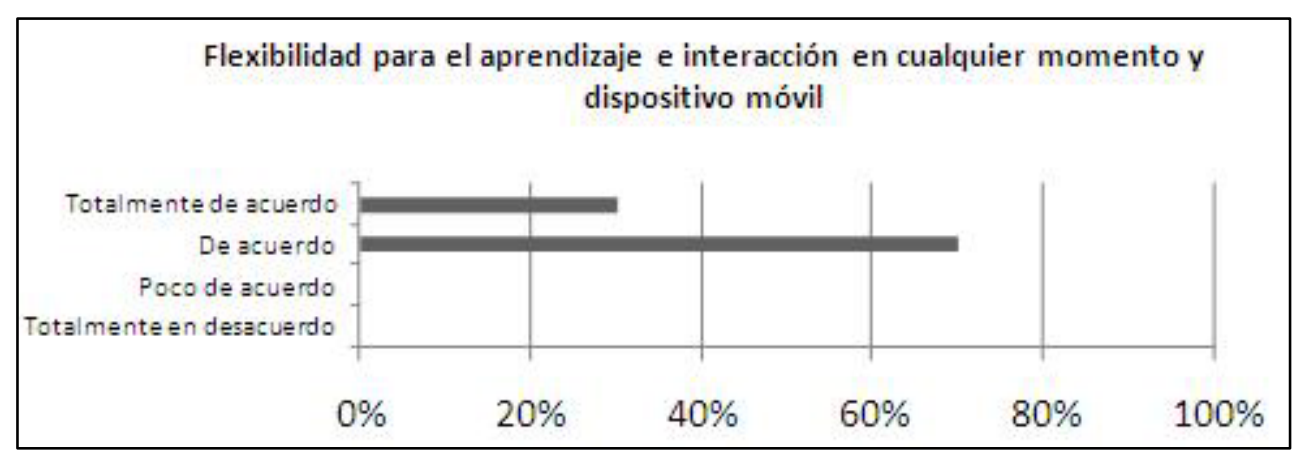

Gráfico 8. Flexibilidad para el aprendizaje e interacción.

La consideración de una utilización provechosa de la plataforma que mejora el proceso de E-A, es un hecho que han captado el 93\% de los alumnos de las distintas materias, Considerando además, un 90\% que la Red social implica el trabajo colaborativo y en $100 \%$ que la integración de las TIC mejora su proceso formativo. 
Vuelve el acuerdo entre el alumnado con un 95\% de apoyo a la idea de que la Red es un instrumento facilitador de innovación y actualización permanente.

La consideración de nuestros alumnos sobre si la experiencia ha producido un trabajo de competencia digital y tratamiento de la información es compartida por el 80\% de ellos. Asimismo, el 79\% de los mismos consideran que se ha promovido el intercambio de opiniones entre los distintos centros que participan en la red. El 85\% de nuestros alumnos creen además que se ha producido un enriquecimiento entre distintas las materias que trabajan de la plataforma, y en este sentido destacan su utilidad para reflejar distintos puntos de vista, para ampliar conocimientos y para enriquecerse.

Las aportaciones de los compañeros son valoradas por la totalidad (100\%) del alumnado, especialmente las opiniones, debates y enlaces a fuentes de información compartidos entre los alumnos.

El 88\% de los discentes consideran que han adquirido conocimientos en el uso de las TIC de manera adecuada. Considerándose casi el $80 \%$ de ellos capaces para integrar este tipo de herramienta en el aula, tal y como podemos ver más profundamente en el siguiente gráfico:

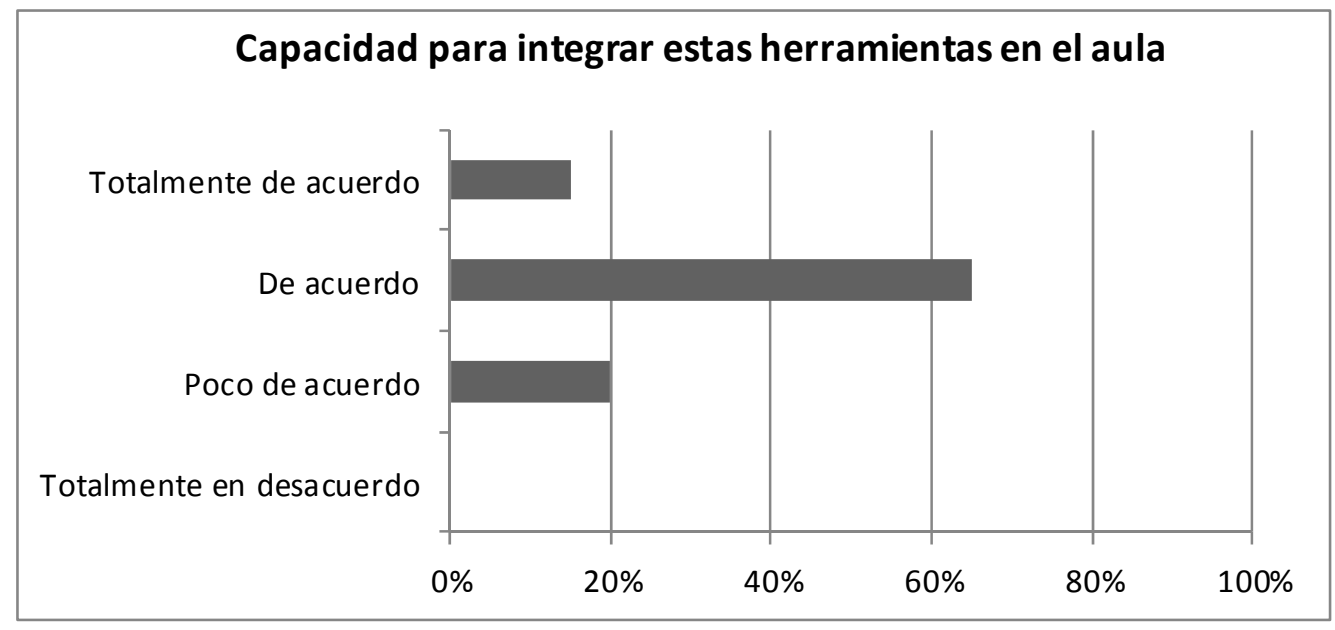

Gráfico 9. Capacidad para integrar herramientas en el aula.

Un aspecto que no se refleja tanto como quisiéramos en el cuestionario es la mejora de la relación profesor-alumno gracias a la red social, ya que un poco más de la mitad (64\%) son los que defienden que sí ocurre mientras un 36\% no consideran que se establezca una mejor relación entre ellos y su docente.

Por otro lado, la actitud positiva hacia el uso de redes es tenida por un $90 \%$ de nuestro alumnado. Asimismo, la red se muestra como facilitadora de contenidos de aprendizaje para el 89\% de los discentes y como motivadora en un 72\% de los casos. 


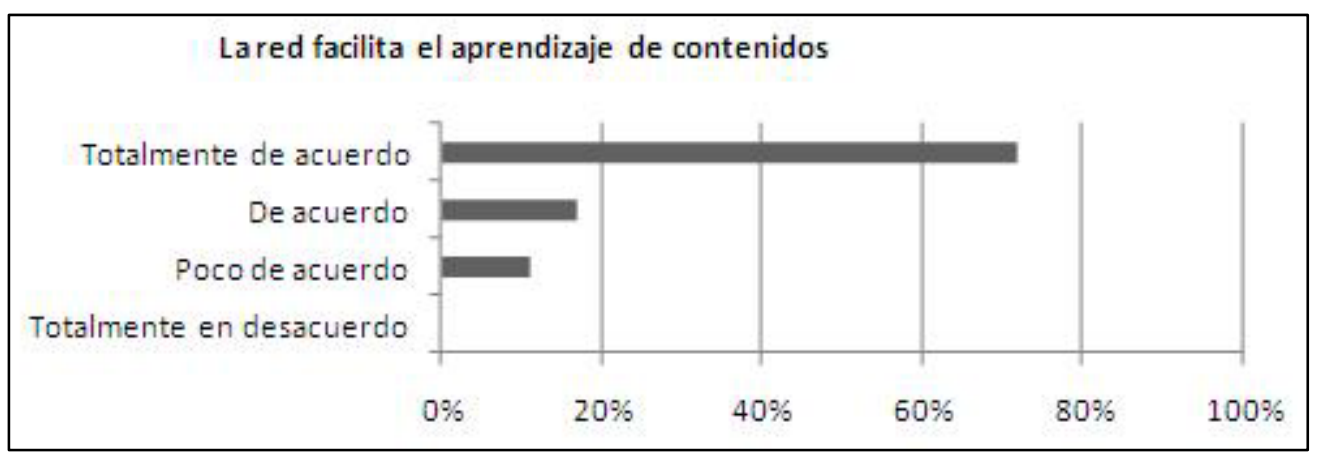

Gráfico 10. La red facilita el aprendizaje de contenidos.

Se muestran a continuación algunos de los comentarios derivados de los instrumentos de corte cualitativo (red social y focus group fundamentalmente), que ayuda a completar el puzzle de las conclusiones:

En relación a utilización y primera toma de contacto con la red UGR/John Moores para la formación. Aspectos generales:

- La red beneficia al alumnado en diversos aspectos utilizando las TIC como la herramienta principal de trabajo y comunicación entre el profesor y los propios alumnos.

- Me parece interesante, innovadora y productiva para nuestra formación.

- Muy interesante y con la que nos será más fácil la compresión de las materias y al mismo tiempo interactuar en tiempo real con compañeros en diferentes zonas geográficas.

- Muy acertada, ya que además de contactar con otros miembros de la plataforma, ayuda a la formación de una manera innovadora que en realidad, motiva al alumnado.

- Rompe con la metodología tradicional 
En relación al grado de aprendizaje de los contenidos de las diferentes materias relacionadas con la organización, gestión y/o administración de Centros Educativos:

- Creo que ha influenciado de manera positiva puesto que con esta metodología hemos llevado un trabajo más autónomo y podemos ampliar nuestros conocimientos de manera independiente

- Es un método innovador, pero no por eso se adquieren menos conocimientos.

- Siempre se aprende más cuando el trabajo es autónomo y puedes dedicarle el tiempo que necesita

- Yo misma marco mi ritmo de trabajo y me preocupo por la búsqueda de información y por mi aprendizaje de los contenidos teóricos.

- El grado de aprendizaje puede haber sido mayor utilizando esta metodología, ya que tenemos que comprender la materia para hacer las preguntas de las guías, en cambio con la metodología tradicional, exclusivamente memorizas los contenidos.

Respecto a la flexibilidad horaria, flexibilidad de contenidos teórico/prácticos y formación a distancia:

- Me parece una gran ventaja, tenemos muchas materias y en ocasiones se nos solapan prácticas y trabajos, esta es una buena oportunidad para tener tiempo.

- Creo que esta es una de las grandes ventajas que tiene esta metodología puesto que muchos de los alumnos no pueden asistir a las clases, y de esta manera tenemos muchas más posibilidades a la hora de distribuir nuestros trabajos.

- Creo que es una de los aspectos más positivos, ya que se adapta a nuestras necesidades y sobretodo, fomenta el trabajo cooperativo.

- Me parece bien, porque el alumno se organiza mejor. En cierto modo, nos hacemos más responsables

- El uso a cualquier hora del material nos facilita mucho más el trabajo de las materias.

Respecto al contacto presencial con el docente:

- Creo que es esencial un seguimiento del profesorado aun con esta forma de enseñanza. El contacto presencial es fundamental.

- Pues la verdad que no lo sé, en principio parece que no hace falta ya que puedes guardar tus dudas para cuando la veas y además siempre está el contacto mediante la plataforma y la red. 


\section{Conclusiones}

Es preciso considerar las conclusiones de este estudio, de forma prudente, puesto que como en cualquier investigación se presentan una serie de limitaciones, especialmente cuando se trata de estudios descriptivos, como éste, donde los resultados e interpretaciones realizadas se centran estrictamente en el contexto particular del campo de estudio abordado (Facultad de Educación de la Universidad de Granada y de la Universidad John Moore de Liverpool), con una idiosincrasia concreta y diferente a otras instituciones o realidades educativas, lo que dificulta e imposibilita, entre otros aspectos, su transferencia o generalización de estas contribuciones. No obstante, sí es una base inicial para realizar en el futuro estudios más amplios, que incluyan mayor número de instituciones, instrumentos y aparataje estadístico para ir conociendo pormenorizadamente el impacto que la acción innovadora, a través de este tipo de metodologías, va incorporando en la enseñanza universitaria.

En función de los resultados obtenidos, podemos concluir que la utilización de redes colaborativas en torno a la integración de plataformas virtuales posibilitan, de manera cierta, la conformación de comunidades de aprendizaje dinámicas y efectivas y redes de conocimiento, redundando todo ello en la calidad de los procesos de E-A. Todo ello sin olvidar, la necesidad de un ejercicio tutorial, más que nunca, reflexivo y comprometido que considere el valor de lo comunitario como imprescindible. Concretamente, con la utilización de la red UGR/John Moores, se ha facilitado el aprendizaje del alumnado, motivándolo, generando y compartiendo sistemáticamente información que ayuda, considerablemente, a la formación de la persona en distintos ámbitos. lo que ha supuesto la conformación de una experiencia en su conjunto muy positivamediante un alto nivel de satisfacción expresado por parte delalumnado. De una formasimilar , otras investigaciones también concluyen en esta misma línea (Santamaría, 2009; Cabero \& Llorente, 2010; Panckhurst \& Marsh, 2011; Trujillo, Cáceres, Hinojo \& Aznar, 2012).

En un primer momento, podemos constatar que no todo el alumnado maneja este tipo de herramientas con facilidad, pero con cierta formación específica, concluimos que las ventajas que aporta la utilización de redes sociales colaborativas está presente, la actitud del alumnado es más positiva, creándose lazos de comunicación e información continua que prevalece una vez concluida la materia. Dichas redes se fortalecen con el propio uso y conocimiento de herramientas afines, potenciándose la creación de entornos personales de aprendizaje que en última instancia se conforman como potenciales para la investigación, la enseñanza flexible y el aprendizaje abierto (Salinas, 2013), contribuyendo a que el alumnado decida sus propias finalidades de aprendizaje auto-gestionándose, comunicándose de manera permanente y todo en pro de la mejora de la calidad, el desarrollo de competencias y la consecución para el logro de objetivos.

En definitiva, la mayor parte de los estudiantes coinciden en señalar la acción innovadora con el uso de las TIC, como una propuesta de aprendizaje enriquecedora basada en una metodología alternativa a la tradicional, que les permite marcar su propio ritmo de aprendizaje, accesible y en constante contacto y comunicación con los grupos. En este sentido, valoran, por una parte, la red social como una oportunidad 
cierta para su desarrollo académico y personal; por otraconsideran que el uso de la plataforma favorece la cooperación y comunicación entre los estudiantes, tanto en los participantes en el proceso como en los contenidos; se comparten experiencias de aprendizaje entre Universidades, valorando la web social y sus oportunidades; favorece el dominio y conocimiento de las TIC (alfabetización digital) como futuros docentes desarrollando la competencia digital y la autonomía e iniciativa personal y aprender a aprender. En esta misma línea se observa una percepción positiva respecto al uso e integración de las TIC en el ámbito docente y la relevancia necesaria de construir entornos de aprendizaje que engloben herramientas y personas- A su vez, aparecen mayores vías de comunicación para la gestión del conocimiento, desde la motivación y el compromiso comunitario, como uno de los principales retos formativos hacia los que la Educación Superior deberá ir trabajando, según las exigencias y demandas actuales.

\section{Referencias bibliográficas}

ALONSO, H. \& LÓPEZ, I. (2008). Adaptando asignaturas al EEES: el caso de Teoría y Técnica de la Publicidad. En I. Rodríguez (Ed.). El nuevo perfil del profesor universitario en el EEES. Claves para la renovación metodológica. Valladolid: Universidad Europea Miguel de Cervantes.

CABERO, J. \& MARÍN, V. (2014). Posibilidades educativas de las redes sociales y el trabajo en grupo. Percepciones de los alumnos universitarios. Comunicar, 42. On Line. Paper.

CÁCERES, M.P. \& AZNAR, I. (2005). Instructive Leadership and the formative role in european network for quality assurance”. The International Journal of Learning, 13 (3), 203-212.

CÁCERES, Ma. P., HINOJO LUCENA, F. J., AZNAR DÍAZ, I. (2011). Incorporación de las TICs en el período escolar de 0 a 6 años: diseño de una entrevista para evaluar las percepciones de los maestros. Revista Pixel Bit, Revista de Medios y Educación, 39, 7-19.

CAMACHO M. (2010). Las redes sociales para enseñar y aprender. Reflexiones pedagógicas básicas. En L. Castañeda (coord). Aprendizaje con redes sociales. Tejidos educativos para los nuevos entornos. Sevilla: Editorial MAD.

CASTAÑEDA, L. \& GUTIÉRREZ, I. (2010). Redes sociales y otros tejidos online para conectar personas. En L. Castañeda (coord). Aprendizaje con redes sociales. Tejidos educativos para los nuevos entornos. Sevilla: Editorial MAD.

COBO, C. \& MORAVEC, J. (2011). Aprendizaje Invisible. Hacia una nueva ecología de la educación. Col-lecció Transmedia XXI. Barcelona: Laboratori de Mitjans Interactius/Publicacions i Edicions de la Universitat de Barcelona.

COMER, J. (1993). School power: implications of an intervention Project. New York: Simon and Shuster. 
DE- GOUVEIA, L. (2012). Comunidades virtuales y el aprendizaje estratégico de cálculo en ingeniería. Pixel Bit, 40, 101-113.

GARCÍA-VALCÁRCEL, A. (2013). Las implicaciones educativas de las redes sociales. En I. Aguaded y J. Cabero (Coords). Tecnologías y medios para la educación en la E-sociedad. Madrid: Alianza Editorial.

GEA, M. \& MONTES, R. (2013). abiertaUGR, la formación abierta basada en Comunidades on line de aprendizaje. En SCOPEO, informe $n^{\circ}$ 2, MOOC. Estado de la situación actual, posibilidades, retos y futuro, 122-138.

GUTIERREZ, A. \& FREIRE, J. (2013). Manifiesto Crowd: La empresa y la inteligencia de las multitudes. Laboratorio de Tendencias.

HARADA, V., LUM, D., \& SOUZA, K. (2003). Building a learning community. Childhood Education, 79 (2), 66-72.

HINOJO-LUCENA, F.J. \& FERNÁNDEZ MARTÍN, F.D. (2002). Diseño de escalas de actitudes para la formación del profesorado en Tecnologías. Comunicar, 19 (octubre), 120-125.

JUBANY, J. (2012). Aprendizaje social y personalizado: conectarse para aprender. Barcelona: Editorial UOC.

KIMBLE, C., HILDRETH, P. \& BOURDON, I. (2008). Communities of Practice: Creating Learning Environments for Educator, 1 (2). Chorlotte, N.C.: Information Age Publishing.

LAURILLARD, D. (2010). Effective Use of Technology in Teaching and Learning in HE. International Encyclopedia of Education. Vol. 4, 419-426.

MAIZ, I. \& TEJADA, E. (2013). La utilización de las redes sociales desde una perspectiva educativa. En J. Barroso y J. Cabero (coords). Nuevos escenarios digitales. Las tecnologías de la información y la comunicación aplicadas a la formación y desarrollo curricular. Madrid: Pirámide.

MARÍN, V., VÁZQUEZ, A. \& CABERO, J. (2012). DIPRO 2.0. Una red social al servicio del profesorado. En Comunicación y Pedagogía. Nuevas Tecnologías y Recursos didácticos. Vol. 261-262, 47-50.

MARQUÉS, L. (2011). La creación de una comunidad de aprendizaje en una experiencia de blended learning. Pixel Bit, 29, 55-68.

MARTÍN MARTÍN, Q. (1999). Paquetes estadísticos SPSS. Madrid: Hespérides.

MARTÍNEZ, F. \& SOLANO, I. (2010). Analizando redes sociales e la Educación Secundaria. En L. Castañeda (coord). Aprendizaje con redes sociales. Tejidos educativos para los nuevos entornos. Sevilla: MAD.

PALLOFF, R.M. \& PRATT, K. (2007). Building Online Learning Communities: Effective Strategies for the Virtual Classroom (2 $2^{\circ}$ ed.). San Francisco: Jossey-Bass. 
READ, T. (2013). Open education for global anotences: The example of UNED. Session: New methods, new pedagogies, virtual and on line education in the framework education reform Project: Cross-Border Education. Madrid, 22-24th.

RODRÍGUEZ ILLERA, J.L. (2008). Comunidades virtuales de práctica y de aprendizaje. Barcelona: Publicacions i Edicions UB.

SALINAS, J. (2013). Enseñanza Flexible y Aprendizaje Abierto, Fundamentos clave de los PLEs. En L. Castañeda y J. Adell (Eds.), Entornos Personales de Aprendizaje: Claves para el ecosistema educativo en red, 53-70. Alcoy: Marfil.

SANTAMARÍA, F. (2009). Posibilidades pedagógicas. Redes sociales y comunidades educativas. TELOS. Cuadernos de comunicación e innovación, 76. Madrid: Fundación Telefónica.

TRUJILLO, J. M. (2013). Redes sociales y educación. En J. Sánchez y J. Ruíz (coords). Recursos didácticos y tecnológicos en educación. Barcelona: Síntesis.

TRUJILlO, J. M., CÁCERES, M. P., HINOJO-LUCENA, F. \& AZNAR, I. (2011). Aprendizaje cooperativo en entornos virtuales. El proyecto redes educativas interuniversitarias. Educar. Universidad Autónoma de Barcelona (UAB), nº 47, 95119.

TRUJILLO, J.M. \& HINOJO-LUCENA, F.J. (2011). Apropiación de recursos y estrategias 2.0 para la innovación educativa en la docencia universitaria. Enseñanza \& Teaching, 28 (2), 61-77.

TRUJILLO, J.M., CÁCERES, M.P., HINOJO-LUCENA, F.J. \& AZNAR, I. (2009). Las redes como elementos constitutivos esenciales en las nuevas organizaciones educativas: El plan escuela TIC 2.0 como apuesta para su desarrollo. Revista de Ciencias de la Educación, 219, 287-310.

VALVERDE, J. (2011). Docentes e-competentes. Buenas prácticas educativas con TIC. Barcelona: Octaedro.

\section{Referencias digitales}

AZNAR, I., CÁCERES, M.P. \& HINOJO-LUCENA, F.J. (5 de junio de 2005). El impacto de las TICs en la sociedad del milenio: nuevas exigencias de los sistemas educativos ante la "alfabetización tecnológica". Etic@net, 4, [formato pdf] http://www.ugr.es/ sevimeco/revistaeticanet/Numero4/Articulos/Formateados/ELI MPACTO.pdf (Consultado 10 de diciembre de 2013)

CABERO, J. \& LLORENTE, M. (15 de octubre de 2010). Comunidades virtuales para el aprendizaje. EDUTEC. Revista electrónica de Tecnología Educativa. Núm. 34 / Diciembre 2010 [formato pdf] http://edutec.rediris.es/Re20 velec2/Revelec34/pdf/Edutec-e_n34_Cabero_Llorente.pdf (Consultado el 11 de noviembre de 2013). 
PANCKHURST, R. \& MARSH, D. (23 de septiembre 2011). Utilización de redes sociales para la práctica pedagógica en la enseñanza superior impartida en Francia: perspectivas del educador y del estudiante. En: El impacto de las redes sociales en la enseñanza y el aprendizaje [monográfico en línea]. Revista de Universidad y Sociedad del Conocimiento (RUSC). Vol. 8, n. ${ }^{\circ} 1,233-252$. UOC [formato pdf] http://rusc.uoc.edu/ojs/index.php/rusc/article/view/v8n1-panckhurst-marsh/v8n1panckhurst-marsh (Consultado 2 de diciembre de 2013).

SOLANO, I. M. (05 de enero de 2006). Metodología de trabajo colaborativo en red: herramientas (weblogs, wikis, webquest y portafolio electrónico) [formato pdf] http://www.ciedhumano.org/files/CongresoEDUTEC05/CONGRESOEDUTEC05I PUBL.html (Consultado 5 de julio de 2007).

THELWALL, M. (21 de febrero de 2008). How are Social Network Sites Embedded in the web? An exploratory link analysis. International Journal of Scientometrics, Informetrics, and Bibliometrics, 12 (1) [formato pdf] http://www.cindoc.csic.es/cybermetrics/articles/v12i1p1.html (Consultado 8 de septiembre de 2010).

\section{Correspondencia con los autores}

Juan Manuel TRUJILLO TORRES

Departamento de Didáctica y Organización Escolar

Facultad de Ciencias de la Educación. Universidad de Granada

Campus Universitario de Cartuja s/n.

18071 Granada

e-mail: jttorres@ugr.es

Inmaculada AZNAR DÍAZ

Departamento de Didáctica y Organización Escolar

Facultad de Ciencias de la Educación. Universidad de Granada

Campus Universitario de Cartuja s/n.

18071 Granada

e-mail: iaznar@ugr.es

María Pilar CÁCERES RECHE

Departamento de Didáctica y Organización Escolar

Facultad de Ciencias de la Educación. Universidad de Granada

Campus Universitario de Cartuja s/n.

18071 Granada

e-mail: caceres@ugr.es 